\title{
Occupational and Environmental Medicine
}

\section{Editorial}

\section{New instructions for authors}

In this issue of Occupational and Environmental Medicine the instructions for authors (see inside front cover) have been changed. Mostly this is just to update them and remove inaccuracies, but there are several important changes. We now require authors to submit a structured abstract with their paper. This makes it easier for readers to grasp the main points of a paper rapidly. Hopefully, it also catches their interest and encourages them to read the whole paper. Together with the structured abstract, we ask authors to give us keywords or key terms to improve indexing of their paper (see the second editorial in this issue).

Another change is that more emphasis is being placed on the importance of work submitted being original and not already published elsewhere. Authors are expected to inform the journal about prior publication of any part of the submitted work, by themselves or others. They should provide a copy of the previous publication and the final judgement about the degree of duplication will be made by the Editor. This requirement to declare previous publication of part of submitted work is one way that Occupational and Environmental Medicine is tackling the increasing problem of duplicate publication. We also rely on the vigilance of expert reviewers, who are now specifically asked if they know of any prior publication of data in submitted papers.

Authors submitting a paper to Occupational and Environmental Medicine are now asked to suggest the names and addresses of three independent people who they consider would be suitable to review their work. The fournal usually sends out papers to two reviewers; one or both of these may be from among those suggested by the authors. The experience of editors of other journals is that reviewers selected by authors are not more lenient than reviewers selected by the editor and that they tend to give good, detailed reviews. Another advantage is that new reviewers are added to the fournal's list, particularly from outside the United Kingdom. This corrects any parochial tendencies and is in line with the international nature of the fournal. Confidentiality of reviewers is preserved by asking for three names and by the uncertainty about whether they will be approached or not. Reviewers are already asked not to discuss the papers they are sent, including with the authors. We will keep the system under review during the next year and will report on its use and effects.

\section{Rejected manuscripts}

From February 1994, authors whose submitted articles are rejected will be advised of the decision and one copy of the article, together with any reviewers' comments, will be returned to them. The fournal will destroy remaining copies of the article but correspondence and reviewers' comments will be kept. 